\title{
THYMIC CARCINOMA - A RARE CASE REPORT
}

\section{NIDHI BANSAL ${ }^{1}$, ARNAV KR ROYCHOUDHURY ${ }^{2}$, HARSHI DHINGRA ${ }^{2}$}

\author{
${ }^{1}$ Department of Immunohematology and Blood Transfusion, GGS Medical \\ College, Faridkot, India \\ ${ }^{2}$ Department of Pathology, ADESH Institute of Medical Sciences and Research, \\ Bathinda, India
}

\begin{abstract}
Introduction. Thymic carcinoma is a rare, invasive mediastinal neoplasm with a tendency to metastasize. They constitute a heterogeneous group of tumors that present differently in terms of both behavior and prognosis.

Case report. We present a case of thymic carcinoma in a 55-year-old male patient known to suffer from Myasthenia gravis, whose chief complaints were fatigability, ptosis and coughing. All electromyography studies were done along with excision biopsy to reach the definitive diagnosis.

Results and conclusion. The histopathological diagnosis of Thymic carcinomaLymphoepithelial variant was established. Though no definite staging system exists for the thymic carcinoma, prognosis of the patient was ascertained by using WeissferdtMoran system, Masaoka and Tsuchiya TNM staging systems. Involvement of the pleural and the pericardial structures leads to poor prognosis, though no distant metastases or lymph nodal metastasis were evident.
\end{abstract} Masaoka

Keywords: thymic carcinoma, lymphoepithelial, mediastinal neoplasms,

\section{Introduction}

The thymus gland is located behind the sternum anterior to the great vessels and reaches its maximum weight at puberty, then gradually undergoes involution. The thymus arises embryologically from the third pharyngeal pouch and branchial cleft on each side. The thymic masses from each side move towards each other and migrate from midline to the anterior mediastinum. Failure of the thymic gland to migrate during embryogenesis leads to the formation of ectopic thymic tissue. Thymic carcinoma is a rare and invasive mediastinal neoplasm that often shows capsular invasion and metastasis. It constitutes a heterogeneous group of tumors that display different biologic behavior and prognosis. Thymic carcinomas have been reported to account for only $0.06 \%$ of all thymic neoplasms [1]. The histological classification of thymic carcinoma was proposed by Levine and Rosai and revised by Suster and Rosai [2]. Histologically the different

Manuscript received: 05.09.2017

Received in revised form: 29.09.2017

Accepted: 12.10.2017

Address for correspondence: drarnav2007@gmail.com variants of thymic carcinoma described are: Squamous cell carcinoma, Lymphoepithelioma-like carcinoma, basaloid carcinoma, mucoepidermoid carcinoma, clear cell carcinoma, sarcomatoid carcinoma and mixed small cell undifferentiated. Most patients with thymic carcinomas are aged between 40 and 60 years [3]. Ninety percent of the thymomas and thymic carcinoma occur in the anterior mediastinum [4]. Typical clinical symptoms and signs that are indicative of anterior mediastinal mass effects include coughing, chest pain and signs of upper airway congestion. Patients often present with advanced disease, the 5-year survival rate being $30-50 \%$ [5]. The largest clinical report by Blumberg et al. [6] showed that innominate vessel invasion was the only prognostic factor and that Masaoka stages could not predict prognosis.

\section{Case report}

A 55 year old male, a known case of Myasthenia gravis, presented to the outpatient department of MGM hospital with complaints of difficulty in swallowing and cough for 3 days, along with bilateral ptosis and progressive easy fatigability for the past 6 months. General 
examinations revealed all vitals within normal range. Hematological and biochemical results were within normal range. High resolution computed tomography showed central necrosis in a large tumor mass measuring $3 \times 5 \mathrm{~cm}$ in the anterior mediastinum which was encroaching the ascending aorta along with fibrosis and early bronchiectatic changes. Nerve conduction studies showed decremental response in abductor pollicis brevis, orbicularis oculi and nasalis muscles. Acetyl choline receptor antibodies serum test showed very high value- $13.20 \mathrm{nMol} / \mathrm{L}$. The thymus mass resected was sent for histopathological examination in $10 \%$ formalin solution.

Gross: We received a firm to hard, grey brown to tan tissue mass measuring $3.8 \times 2.8 \times 1 \mathrm{~cm}$ along with pericardium measuring $16 \times 12 \times 1 \mathrm{~cm}$ and cut piece of lung measuring $2.6 \times 2 \times 1.6 \mathrm{~cm}$. Cut section was gritty with greyish white areas with areas of hemorrhage (Figure 1). After routine processing the sections were stained with Hematoxylin Eosin.

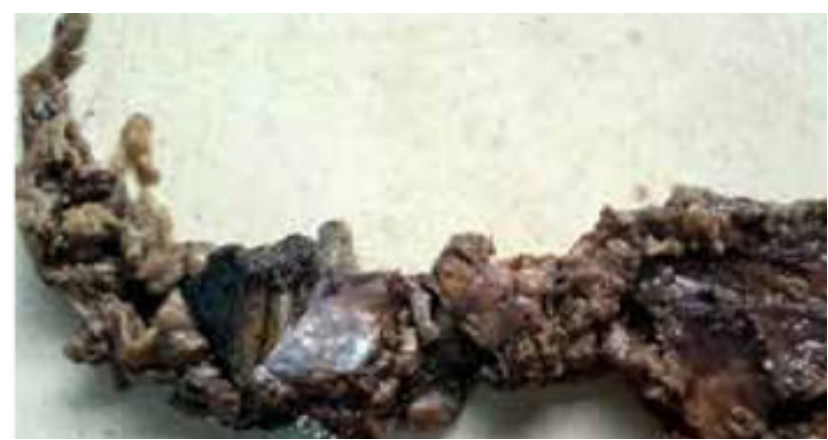

Figure 1. Gross picture showing solid tumor with adjacent pericardium and pleura.

Microscopy: H E stained sections (Figure 2) show cohesive cellular growth arranged in a syncytial pattern. Individual tumor cells show regularly round to oval indistinct nuclear outlines with vesicular nucleus and eosinophilic nucleoli. Abundant geographic necrosis and abortive Hassall's corpuscles, rosettes admixed with dense inflammatory infiltrate comprising of lymphocytes.

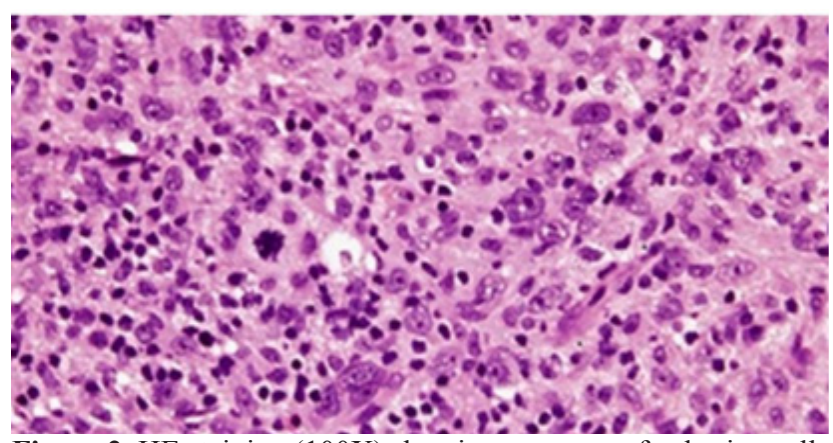

Figure 2. HE staining (100X) showing presence of cohesive cells in syncytial pattern with vesicular round nucleus and prominent nucleoli along with abundance infiltration of lymphocytic infiltrate and presence of atypical mitotic figures.

\section{Discussion}

Thymic carcinomas account for approximately $15 \%$ of all the primary thymic epithelial tumors. Surgical excision is done for both diagnostic as well as therapeutic purposes. Thymic carcinomas are rare neoplasms and because of their aggressive nature, they are likely to produce hematogenous and lymphatic spread, locally and distally. The diagnosis of thymic epithelial neoplasm has been a topic of controversy for many years.

Chung et al. reported 305 patients with thymic carcinoma compiled from a Medline search from 1966 to 2000 [7]. Most of these patients were asymptomatic or had developed late symptoms and were treated in the late stages of the disease. Reports about the clinical prognostic factors and treatment of choice are rare as most of the series had very limited number of patients.

Kondo et al. [8] reported 1320 patients with thymic epithelial tumors who were treated in 115 institutes in Japan from 1990 to 1994 . Their report included 186 thymic carcinomas. Other than these reports from multiple institutions, only limited number of patients have ever been analyzed. Some reports suggested that tumor resectability, staging and histological grading influenced the outcome. Nevertheless, the clinicopathologic characteristics are still unclear. Reasons for this being the lack of predictive value associated with the morphology of these tumors.

Local regional invasion of nearby organs by thymic carcinoma is very common, lung being the commonest. Our case showed tumor invasion of the aorta and was one of the major determinants of prognosis, which was similar to the result reported by Blumberg et al [6]. Thymic carcinoma with aortic or pulmonary vessel involvement always requires complete resection, not only because it extensively encases the great vessels, but also because distant metastasis are frequently noted. These patients may have had early distant metastasis because the tumor cells penetrated and spread from the great vessels.

The histological classification of thymic carcinoma has been important because of its prognostic significance. Tumors in high grade histology group are characterized by an aggressive course and high incidence of local recurrence and distant metastases. On the contrary tumors in the low grade histological group are characterized by a comparatively favorable clinical course and a low incidence of local recurrence and distant metastases. WeissferdtMoran [9] also gave the TNM surgical Staging system for Thymic carcinoma (Table I).

Recently, a new classification scheme was introduced by the World Health Organization (WHO) in an attempt to standardize the nomenclature to facilitate the diagnosis of primary thymic epithelial neoplasms [10].

Masaoka staging system is one of the two factors, including completeness of surgical resection, that most strongly correlates with prognosis of thymomas and has been used so far because of the absence of any thymic 
carcinoma specific stage classifications.

We classified our case as Masaoka [11] stage IV4a (Table II) because of the pleural and pericardial dissemination.

According to the Tsuchiya [12] TNM staging system (Table III) it was Stage IV4a because of the pleural and pericardial implantation of tumor with presence of few anterior mediastinal lymph node metastasis, however no distant organ metastasis was seen.

There have been no thymic carcinoma specific stage classifications. It is usually diagnosed with advanced stages.

Table I. Weissferdt-Moran [9] TNM Staging system for Thymic carcinoma.

\begin{tabular}{l|l}
\hline & \multicolumn{1}{|c}{ DESCRIPTION } \\
\hline T1 & Tumor limited to thymic gland \\
\hline T2 & Tumor invading visceral pleura, lung, pericardium, great vessels, chest wall or diaphragm \\
\hline T3 & Direct extra thoracic tumor extension, beyond thoracic inlet or diaphragm \\
\hline N0 & No lymph node metastasis \\
\hline N1 & Lymph node metastasis to intrathoracic lymph nodes \\
\hline M0 & No distant metastasis \\
\hline M1 & Distant metastasis (indirect tumor spread, including metastasis to extra thoracic lymph nodes) \\
\hline Steage I & T1N0M0 \\
\hline Stage II & T2N0M0 \\
\hline Stage III & T3N0M0
\end{tabular}

Table II. Masaoka-Koga staging system of thymoma.

\begin{tabular}{l|l}
\hline STAGE & DESCRIPTION \\
\hline I & Macroscopically completely encapsulated and microscopically no capsular invasion. \\
\hline II & $\begin{array}{l}\text { Macroscopic invasion into surrounding fatty tissue or mediastinal pleura } \\
\text { Microscopic invasion into pleura }\end{array}$ \\
\hline III & Macroscopic invasion into neighboring organs (pericardium, great vessel, or lung) \\
\hline IV & $\begin{array}{l}\text { Pleural or pericardial dissemination } \\
\text { Lymphogenous or hematogenous metastasis }\end{array}$ \\
\hline
\end{tabular}

Table III. Tsuchiya TNM staging for thymic carcinoma.

\begin{tabular}{|c|c|}
\hline & DESCRIPTION \\
\hline Pt1 & Completely encapsulated tumor \\
\hline $\mathrm{Pt} 2$ & $\begin{array}{l}\text { Tumor breaking through capsule, invading thymus or fatty tissue (may be adherent to mediastinal pleura but not invading } \\
\text { neighbouring organs) }\end{array}$ \\
\hline Pt3 & $\begin{array}{l}\text { Tumors breaking through the mediastinal pleura or pericardium or invading neighboring organs such as greater vessels } \\
\text { or lungs. }\end{array}$ \\
\hline Pt4 & Tumor with pleural or pericardial implantation \\
\hline PN0 & No lymph node metastasis \\
\hline PN1 & Metastasis in anterior mediastinal lymph nodes \\
\hline PN2 & Metastasis in intrathoracic lymph nodes, excluding anterior mediastinal lymph nodes \\
\hline PN3 & Metastasis in extra thoracic lymph nodes \\
\hline PM0 & No distant organ metastasis \\
\hline PM1 & With distant organ metastasis \\
\hline Stage I & $\mathrm{T} 1$ or $\mathrm{T} 2 \mathrm{~N} 0 \mathrm{M} 0$ \\
\hline Stage II & $\mathrm{T} 1$ or $\mathrm{T} 2 \mathrm{~N} 1 \mathrm{M} 0$ \\
\hline Stage III & T3 N0 or N1 M0 \\
\hline Stage IVa & T4 N0 or N1 M0 \\
\hline Stage IVb & Any $\mathrm{T}, \mathrm{N} 2$ or $\mathrm{N} 3, \mathrm{M} 0$ \\
\hline Stage IVc & Any $\mathrm{T}$, any $\mathrm{N}, \mathrm{M} 1$ \\
\hline
\end{tabular}




\section{Conclusion}

Complete resection of the thymic tumor is the best way to achieve long term survival, though survival also depends on the lymph node status. It is one of the most significant prognostic factors in thymic carcinoma. The patients with tumor invasion of the great vessels have poor prognosis as they may further lead to distant organ dissemination. Also, it has been seen that preoperative radiotherapy and chemotherapy improves the chances of better resectability of the thymic carcinomas with subsequent long term survival.

\section{References}

1. Greene MA, Malias MA. Aggressive multimodality treatment of invasive thymic carcinoma. J Thorac Cardiovasc Surg. 2003; 125 (2):434-436.

2. Suster S, Rosai J. Thymic carcinoma. A clinicopathologic study of 60 cases. Cancer. 1991;67:1025-1032.

3. Schmidt-Wolf IG, Rockstroh JK, Schüller H, Hirner A, Grohe C, Müller-Hermelink HK, et al. Malignant thymoma: current status of classification and multimodality treatment. Ann Hematol. 2003;82(2):69-76.

4. Cameron RB, Loehrer PJ, Thomas CR Jr. Neoplasms of the mediastinum. In: DeVita VT Jr, Lawrence TS, Rosenberg SA: Cancer: Principles and Practice of Oncology. 9th ed. Philadelphia, Pa: Lippincott Williams \& Wilkins, 2011, pp 871-81.
5. Eng TY, Fuller CD, Jagirdar J, Bains Y, Thomas CR Jr. Thymic carcinoma: state of the art review. Int J Radiat Oncol Biol Phys. 2004;59(3):654-664, 2004.

6. Blumberg D, Burt ME, Bains MS, Downey RJ, Martini N, Rusch V, et al. Thymic carcinoma: current staging does not predict prognosis. J Thorac Cardiovasc Surg. 1998;115:303-308; discussion 308-9.

7. Chung DA. Thymic carcinoma--analysis of nineteen clinicopathological studies. Thorac Cardiovase Surg. 2000;48:114-119.

8. Kondo K, Monden Y. Therapy for thymic epithelial tumors: a clinical study of 1,320 patients from Japan. Ann Thorac Surg. 2003;76:878-884; discussion 884-5.

9. Weissferdt A, Moran CA. Thymic carcinoma, part 2: a clinocopathologic correlation of 33 cases with a proposed staging system. Am J Clin Pathol. 2012;138:115-121.

10. Travis WD, Brambilla E, Muller-Hermelink HK, et al. World Health Oragnization classification of tumours. Pathology and genetics of tumours of the lung, pleura, thymus and heart. Lyon: International Agency for Research on Cancer Press; 2004:146151.

11. Masaoka A, Monden Y, Nakahara K, Tanioka T. Follow-up study of thymomas with special reference to their clinical stages. Cancer. 1981;48:2485-2492.

12. Tsuchiya R, Koga K, Matsuno Y, Mukai K, Shimosato Y. Thymic carcinoma: proposal for pathological TNM and staging. Pathol Int. 1994;44:505-512. 